\title{
Preparing thin cross sections of Arabidopsis roots without embedding
}

\author{
Naoyuki Sotta and Toru Fujiwara \\ Department of Applied Biological Chemistry, Graduate School of \\ Agricultural and Life Sciences, The University of Tokyo, Tokyo, Japan
}

BioTechniques 63:281-283 (December 2017) doi 10.2144/000114621

Keywords: Cross section, root, microscopy, vibratome, Arabidopsis thaliana

Supplementary material for this article is available at www.BioTechniques.com/article/114621.

Here, we describe a method for obtaining thin cross sections of Arabidopsis thaliana roots without fixation and embedding. Roots were grown in pinholes made in a solidified growth medium, and cross sections were prepared without pretreatment. Using this method, we detected unique distributions of two polar-localized proteins green fluorescent protein (GFP)-tagged BOR1 and NIP5;1 - with less sample preparation time than conventional methods. This method is simple, rapid, and yields high-quality cross-section images that are free from artifacts commonly associated with embedding or the sample preparation procedures used in many conventional methods.

The observation of the localization of fluorescently tagged proteins in root tissue is useful for a wide range of plant-related research. Protein localization and/or the polarity of radial (transverse) profiles are often examined. To obtain such profiles, either cross-sectioning of root tissue or three-dimensional (3-D) reconstruction of longitudinal images is required.

For physical cross-sectioning, roots are typically embedded in a supporting medium, such as agarose, paraffin, or resin, since roots are too thin to be handled without support. Embedding often involves heating of the samples, which may introduce artifacts. Before embedding, plants are often killed and treated with a fixative, such as formalin-aceto-alcohol (FAA), to preserve the cells in their natural condition (1). However, chemical fixation is often incompatible with the use of fluorescent proteins. Therefore, non-fixed tissue should ideally be observed over a short time scale to minimize artifacts related to protein localization. 3-D reconstruction is another method for obtaining root cross-sectional images of fluorescent proteins, but even for relatively thin Arabidopsis roots, which have a diameter of 130 $\mu \mathrm{m}$, the inner tissues are too deep for confocal microscopy to obtain clear images for 3-D reconstruction.

Among the commonly used methods, cross-sectioning of roots embedded in agarose gel as the supporting medium is a simple and time-saving technique compared with embedding in resin or paraffin; however, the embedding steps are still artifact-prone. During these steps, the roots are exposed to melted gel, the temperature of which is higher than that of standard plant growth conditionstypically $22^{\circ} \mathrm{C}$ for Arabidopsis thalianaeven if low-melting-temperature agarose is used. In addition, to ensure less damage to tissue sections, it is important for the embedding gel to be sufficiently polymerized. For this reason, some protocols require a gel solidification step at $4^{\circ} \mathrm{C}$ for $3 \mathrm{~h}$ (2). For non-fixed plant tissue, this time-scale and the effects of high or low temperature increase the risk of artifacts.

The method described here minimizes these disadvantages by omitting the artifact-prone gel-embedding steps through the use of plant growth medium as the embedding medium for sectioning. This allows plants to be kept under growing conditions until minutes before observation.

We observed the radial accumulation pattern of the green fluorescent protein (GFP)-tagged $A$. thaliana boron transporters NIP5;1 and BOR1 $(3,4)$ using our new method. These proteins are expressed under low-boron conditions and are known to have a polar localization $(5,6)$. MGRL medium (7) containing $0.3 \mu \mathrm{M}$ boric acid and solidified using 3\% gellan gum was used as the growth/embedding medium. For preparation of precise transverse sections, the roots must grow straight within the gel. To guide root growth, the medium was vertically perforated with sterilized acupuncture needles $100 \mu \mathrm{m}$ in diameter (Seirin, Shizuoka, Japan) (Figure 1A). To guide the germinated root into the pinhole, a pit was made on the top of the pinhole with a sterilized toothpick 1 $\mathrm{mm}$ in diameter (Figure 1B). Seeds were surface-sterilized with $70 \%$ ethanol for 5 min followed by $99.5 \%$ ethanol for $1 \mathrm{~min}$. After sterilization, seeds were placed in the pits (Figure 1C). Plates were kept at $4^{\circ} \mathrm{C}$ for $1-3$ days before incubation in a growth chamber set to $22^{\circ} \mathrm{C}$ under a 16 h light/8 h dark cycle. Approximately 4 days after germination, seedlings with roots growing through the holes (Figure 1D) were selected and cut out from the

\section{METHOD SUMMARY}

Here, we present a method for obtaining fresh, thin cross sections of roots without gel-embedding by allowing the roots to grow into pinholes in growth medium, with the latter acting as the embedding gel. Without the need for any pre-processing, 100-200 $\mu \mathrm{m}$-thick cross sections can be obtained by sectioning the root-containing gel. This procedure allows observation of the localization of fluorescently tagged proteins within a few minutes after the start of root treatment. 
A

B

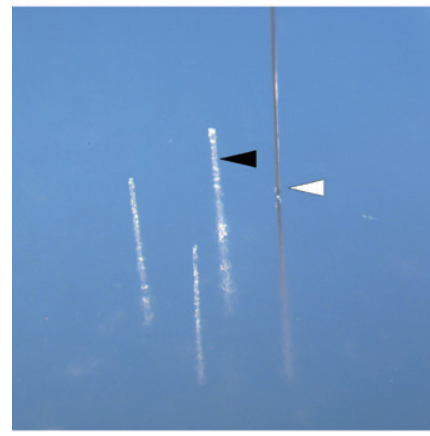

C

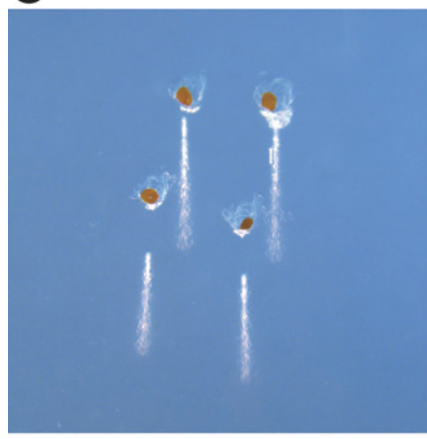

E
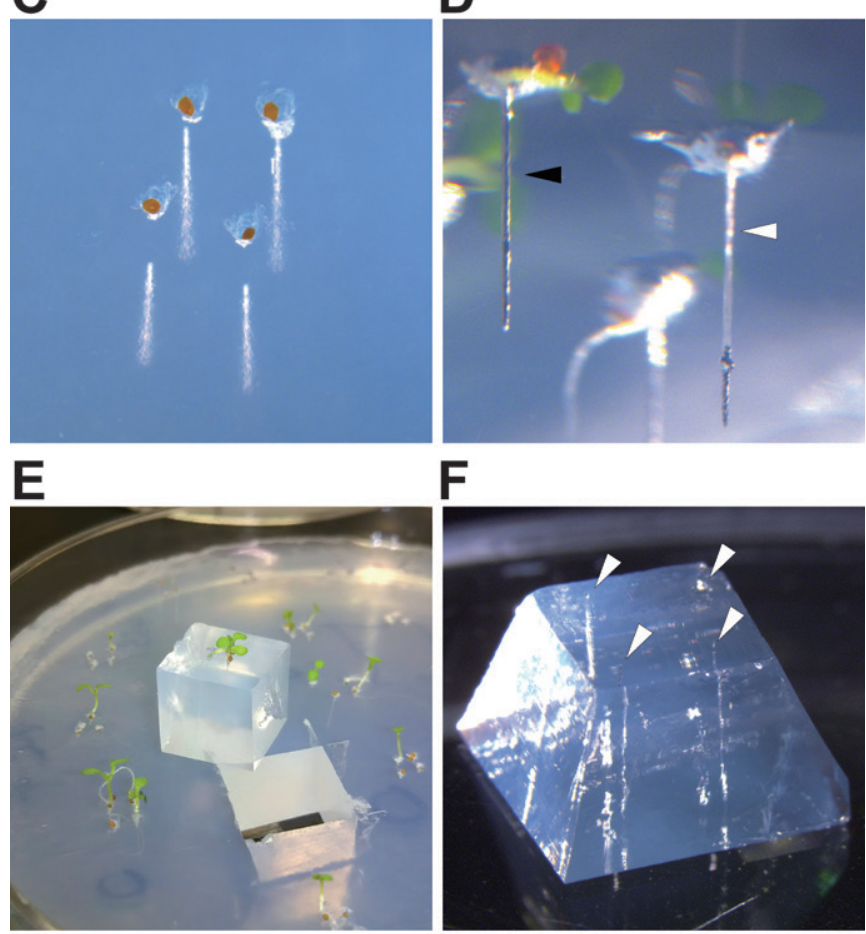

$\mathbf{F}$

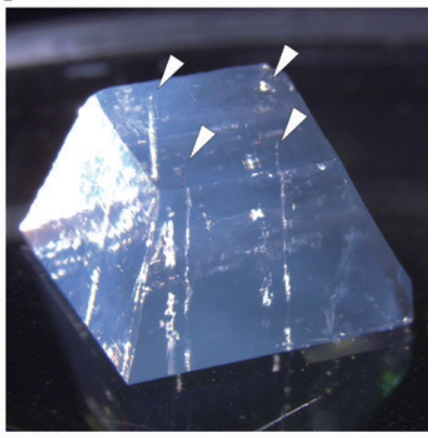

Figure 1. Plant culture and sample preparation for no-embedding cross-sectioning. (A) Perforating a growing/embedding medium plate with an acupuncture needle (100 $\mu \mathrm{m}$ in diameter, white arrowhead) to generate a guide hole for root growth (black arrowhead). (B) Making pits with a toothpick on the top of the pinholes to guide root growth into the holes. (C) Appearance of the samples after sowing. (D) A root successfully growing through the pinhole (white arrowhead) and a hole without a root (black arrowhead). (E) Cutting out seedlings along with the surrounding gel (about $1 \mathrm{~cm} \times 1 \mathrm{~cm}$ ). (F) Samples trimmed for vibratome sectioning. White arrowheads indicate the pinholes and roots. The upper surface of the frustum is $\sim 5 \mathrm{~mm} \times \sim 5 \mathrm{~mm}$.
A

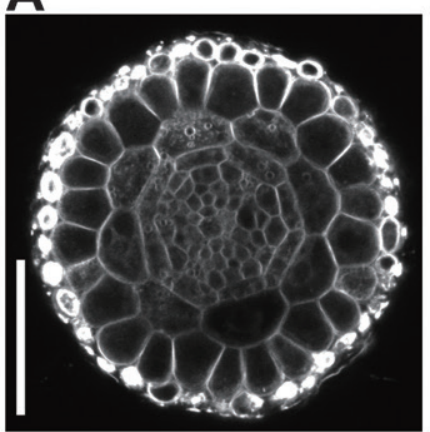

B

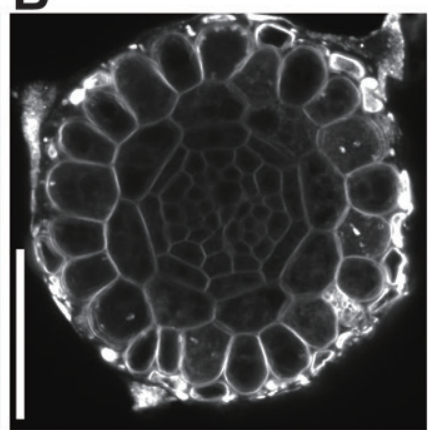

C

D

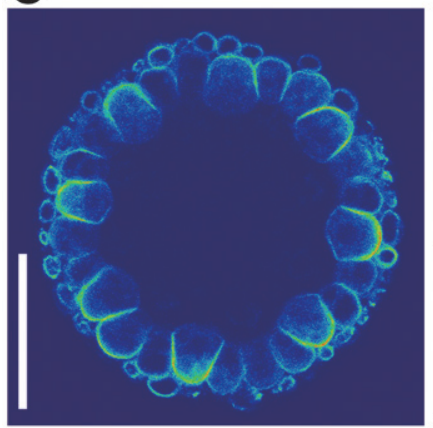

Fluoresence intensity

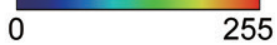

Figure 2. Root cross-section images of GFP-NIP5;1 and BOR1-GFP obtained by no-embedding cross-sectioning. Cross-section images of GFP-NIP5;1. $(A, C)$ and BOR1-GFP (B,D) obtained by confocal microscopy are shown. $(A, B)$ The plasma membrane was visualized by FM4-64 staining. (C,D) Green fluorescent protein (GFP) fluorescence intensity is expressed on a pseudo-color scale. The color scale is shown at the bottom. Bars: $50 \mu \mathrm{m}$.

medium plate, along with the surrounding gel (Figure 1E). The gel was then trimmed into a frustum that was $5-10 \mathrm{~mm}$ on the bottom side (Figure 1F). The trimmed gel was cut into slices $200-\mu$ m-thick with a vibratome (Micro Slicer Zero 1; Dosaka EM, Kyoto, Japan). The cutting bath was filled with PBS $(137 \mathrm{mM} \mathrm{NaCl}, 2.68 \mathrm{mM}$ $\mathrm{KCl}, 10 \mathrm{mM} \mathrm{Na}_{2} \mathrm{HPO}_{4}, 2 \mathrm{mM} \mathrm{KH}_{2} \mathrm{PO}_{4}, \mathrm{pH}$ 7.4), which is critical for observation of GFP as low pH weakens GFP fluorescence (8). The gel slices were collected with a fine-point brush and immediately imaged with a confocal microscope (FV1000; Olympus, Tokyo, Japan). For visualization of the plasma membrane, $4 \mathrm{\mu g} /$ mL FM4-64 (Thermo Fisher, Yokohama, Japan) in PBS was used as the mounting solution for microscopy.

Cross-section images are shown in Figure 2. Inward and outward polarities of BOR1-GFP and GFP-NIP5;1, respectively, were evident in the cross sections (9). In addition, it should be noted that the profile seemed to be free from surface-dependent expression pattern artifacts; this was not necessarily the case in previous reports, in which fluorescence was absent in some parts of the cross sections (10). A. thaliana seedlings are commonly grown on the surface of gel-solidified medium; therefore, some of the root surface is in contact with the medium, while the other side is exposed to the air. This may cause a bias in the expression patterns of proteins for which expression is regulated according 
to the local environment. In our method, as roots grow through the hole, which is slightly narrower than the root diameter, the whole root surface is in contact with the medium, thus ensuring a uniform environment surrounding the roots. Although different growth conditions may cause other artifacts derived from possible differences in growth, no significant difference was detected in root growth after 4 days between plants grown into media $[5.81 \pm 1.28 \mathrm{~mm}$ (mean $\pm \mathrm{SD}), n=57$ ) and plants grown on the surface of medium plates $(5.61 \pm 1.75 \mathrm{~mm}$, $n=24)$ ( $P=0.61$, Welch's $t$-test). In this no-embedding cross-sectioning method, as the roots are maintained in the growing medium until the time of trimming, the interval between the first physical stress and observation by microscopy can be reduced to a few minutes. This not only minimizes artifacts caused by the embedding step, but also decreases sample preparation time by omitting the embedding process. This rapid sample preparation technique renders observation of protein localization in non-fixed root tissue more reliable.

\section{Author contributions}

N.S. designed and performed the experiments and prepared the manuscript. T.F. supervised the study and corrected the manuscript.

\section{Acknowledgments}

This work has been supported by the Japan Society for the Promotion of Science via Grants-in-Aid for Scientific Research No. 25221202 to TF. Seeds of BOR1-GFP bor1-1 (6) and GFP-NIP5;1 (5) plants were provide by Junpei Takano and Mayuki Tanaka, respectively. We thank Yuko Kawara for technical assistance.

\section{Competing interests}

The authors declare no competing interests.

\section{References}

1. Johansen, D.A. 1940. Plant Microtechnique. McGraw-Hill Book Company, London, United Kingdon.

2. Athman, A., S.K. Tanz, V.M. Conn, C. Jordans, G.M. Mayo, W.W. Ng, R.A. Burton, S.J. Conn, and M. Gilliham. 2014. Protocol: a fast and simple in situ PCR method for localising gene expression in plant tissue. Plant Methods 10:29.
3. Takano, J., M. Wada, U. Ludewig, G. Schaaf, N. von Wiren, and T. Fujiwara. 2006. The Arabidopsis major intrinsic protein NIP5;1 is essential for efficient boron uptake and plant development under boron limitation. Plant Cell 18:1498-1509.

4. Takano, J., K. Noguchi, M. Yasumori, and M. Kobayashi. 2002. Arabidopsis boron transporter for xylem loading. Nature 420:337-340

5. Tanaka, M., J. Takano, Y. Chiba, F. Lombardo, Y. Ogasawara, H. Onouchi, S. Naito, and T. Fujiwara. 2011. Borondependent degradation of NIP5;1 mRNA for acclimation to excess boron conditions in Arabidopsis. Plant Cell 23:3547-3559.

6. Takano, J., K. Miwa, L. Yuan, N. von Wiren, and T. Fujiwara. 2005. Endocytosis and degradation of BOR1, a boron transporter of Arabidopsis thaliana, regulated by boron availability. Proc. Natl. Acad. Sci. USA 102:12276-12281.

7. Fujiwara, T., M.Y. Hirai, M. Chino, Y. Komeda, and S. Naito. 1992. Effects of sulfur nutrition on expression of the soybean seed storage protein genes in transgenic petunia. Plant Physiol. 99:263-268.

8. Doherty, G.P., K. Bailey, and P.J. Lewis. 2010. Stage-specific fluorescence intensity of GFP and mCherry during sporulation in Bacillus subtilis. BMC Res. Notes 3:303.

9. Takano, J., M. Tanaka, A. Toyoda, K. Miwa, K. Kasai, K. Fuji, H. Onouchi, S. Naito, and T. Fujiwara. 2010. Polar localization and degradation of Arabidopsis boron transporters through distinct trafficking pathways. Proc. Natl. Acad. Sci. USA 107:5220-5225.

10. Wang, S., A. Yoshinari, T. Shimada, I. Hara-Nishimura, N. Mitani-Ueno, J. Feng Ma, S. Naito, and J. Takano. 2017. Polar Localization of the NIP5;1 Boric Acid Channel Is Maintained by Endocytosis and Facilitates Boron Transport in Arabidopsis Roots. Plant Cell 29:824-842.

Received 04 September 2017; accepted 01 November 2017.

Address correspondence to Toru Fujiwara, Department of Applied Biological Chemistry, Graduate School of Agricultural and Life Sciences, The University of Tokyo 1-1-1 Yayoi, Bunkyo-ku, Tokyo, Japan 113-8657. E-mail: atorufu@mail.ecc.u-tokyo.ac.jp

To purchase reprints of this article, contact: biotechniques@fosterprinting.com

\section{always on and always at temperature}

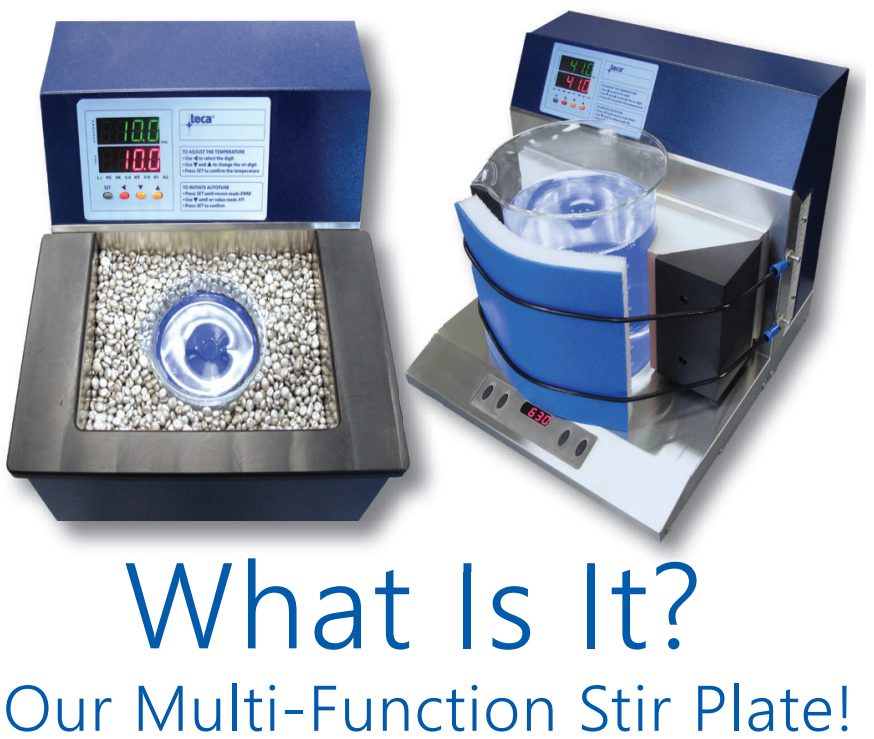

$\checkmark$ cool \& heat samples $\sqrt{ }$ stir mode maintains solution mix

$\checkmark$ bead bath accessory accepts various sized vessels; use as a soaking or stirring bath - no ice! (left)

$\checkmark$ sleeve accessory accepts range of beaker sizes to deliver direct \& quick temperature control (right)

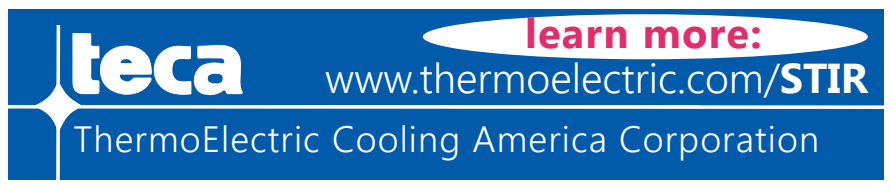

